\title{
Gammapy: high level data analysis for extragalactic science cases with the Cherenkov Telescope Array
}

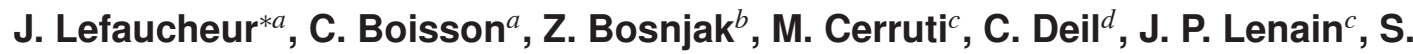 \\ Pita $^{e}$ and A. Zech ${ }^{a}$ for the CTA consortium \\ ${ }^{a}$ LUTH, Observatoire de Paris, PSL Research University, CNRS, Université Paris Diderot \\ 5 Place Jules Janssen, 92190 Meudon, France \\ ${ }^{b}$ Faculty of Electrical Engineering and Computing \\ University of Zagreb, 10000 Zagreb, Croatia \\ ${ }^{c}$ Laboratoire de Physique Nucléaire et de Hautes Energies (LPNHE) \\ 4 place Jussieu, F-75252, Paris Cedex 5, France \\ ${ }^{d}$ Max-Planck-Institut für Kernphysik \\ P.O. Box 103980, D 69029 Heidelberg, Germany \\ ${ }^{e}$ APC, AstroParticule et Cosmologie, Université Paris Diderot, CNRS/IN2P3, CEA/Irfu, \\ Observatoire de Paris, Sorbonne Paris Cité \\ 10, rue Alice Domon et Léonie Duquet, 75205 Paris Cedex 13, France \\ E-mail: julien.lefaucheur@obspm.fr
}

The Cherenkov Telescope Array (CTA) observatory will probe the non-thermal universe above $20 \mathrm{GeV}$ up to several hundreds of $\mathrm{TeV}$ with a significant improvement in sensitivity and angular resolution compared to current experiments. Its outstanding capabilities will allow to increase the number of extragalactic cosmic accelerators detected at very high energy (VHE) and therefore to better constrain the population of VHE accelerators and the gamma-ray absorption processes in the intergalactic medium. For the first time in the history of imaging atmospheric Cherenkov telescopes (IACTs), CTA will be an open observatory and high-level data will be made available to the astronomical community. Gammapy is an open-source Python package developed by the Cherenkov telescope community that provides tools to simulate the gamma-ray sky and analyse IACT data. The versatile architecture of, and steady user contributions to Gammapy enable a large variety of high-level data analyses. Examples of Gammapy applications are presented, particularly in the context of extragalactic science with CTA.

35th International Cosmic Ray Conference - ICRC2017

10-20 July, 2017

Bexco, Busan, Korea

\footnotetext{
*Speaker.
} 


\section{Introduction}

The next generation of Imaging Atmospheric Cherenkov Telescopes, the Cherenkov Telescope Array, will probe the $\gamma$-ray sky above $20 \mathrm{GeV}$ with an unmatched sensitivity and angular resolution using more than 100 telescopes distributed on the South (Chile) and on the North (La Palma) sites. To reach this goal, three different types of telescopes will be used : the $23 \mathrm{~m}$ diameter Large-Size Telescopes (LST), the $12 \mathrm{~m}$ diameter Medium-Size Telescopes (MST) and the $4 \mathrm{~m}$ diameter SmallSize Telescopes (SST). They are respectively adapted to detect photons of $20 \mathrm{GeV}-200 \mathrm{GeV}$ (field of fiew of $\sim 4^{\circ}$ ), $0.1 \mathrm{TeV}-10 \mathrm{TeV}$ (field of fiew of $\sim 7^{\circ}$ ) and $1 \mathrm{TeV}-300 \mathrm{TeV}$ (field of fiew of $\sim 9^{\circ}$ ).

With its improved sensitivity and higher field of view compared to current experiments, namely MAGIC, H.E.S.S. and VERITAS, CTA will improve our current knowledge on active galactic nuclei (AGN) and especially on blazars. Nowadays, population studies are quite limited by the low/biased sample of blazars detected at very high energy $(E \geq 100 \mathrm{GeV})$. The high quality spectral resolution of CTA will help to look for hadronic signatures in AGN spectra [1]. Furthermore, it will also help to better constrain the intergalactic medium opacity to $\gamma$-rays, and especially the flux density of the diffuse extragalactic background light (EBL). The first VHE detection of $\gamma$-ray bursts (GRBs) might shed light on the radiative processes and the particle content in those accelerators and might provide a new class of distant objects to constrain the EBL density.

CTA is expected to operate for thirty years and unlike current experiments it will be an open observatory, meaning that high level data, such as event lists and instrument response functions (IRFs), will be made available to the scientific community. Gammapy ${ }^{1}$ is a community-developed, open-source Python package for $\gamma$-ray astronomy [2]. It is built on widely-used scientific packages (Astropy, Numpy, Scipy) and provides tools to simulate and analyse IACT data. It was thought to produce high level data, such as spectra, maps, light curves and even catalogues, taking as inputs lower level data such as event lists and instrument response functions. Classical methods to analyse data in very high energy astronomy, such as background substraction or full-forward folding spectral reconstruction are implemented and were highly tested with real data, especially in the H.E.S.S. experiment. Cube-style analysis, in which a spectral and a spatial model are jointly adjusted, is also implemented [3].

In this contribution, we show how Gammapy can easily be used to tackle some extragalactic science cases of CTA. We first present in Section 2 how point-like source simulations are made within Gammapy. Section 3 describes how it can contribute to the ongoing efforts to estimate the number of expected blazars that could be detected with CTA. Section 4 is a technical discussion on how Gammapy can be used to constrain the EBL scale factor. Section 5 briefly presents an application about GRB detectability. The Python scripts used to produce all results and figures presented here are available at https://github.com/gammapy/icrc2017-gammapy-cta-egal.

\section{Simulations}

In the following, we will focus on point-like source simulations. To reach this goal, simulations are generated with a set of instrument response functions, which are produced by the simulation group in the consortium [4], including :

\footnotetext{
${ }^{1}$ http://docs.gammapy.org/en/latest/
} 
- effective area as function of true energy,

- background rate as function of reconstructed energy,

- energy migration matrix from true to reconstructed energy

An energy dependent-angular cut is applied to maximise the significance in each interval of reconstructed energy. To compute an expected excess for a given spectral models and exposure we multiply the desired input spectra with the absorption of one of the available EBL model $[5,6,7]$ in Gammapy. We sum the randomised expected excess and background counts to get the data in the ON region of the source. Finally, we randomised the background counts multiplied by the normalisation between the $\mathrm{ON}$ and the OFF regions, taken as 0.2 in the following, to get the data in the OFF region. The average time to run 100 simulations is less than $900 \mathrm{~ms}$ on a personal computer ${ }^{2}$. In the following sections, the level of significance is estimated with the formula 17 of $\mathrm{Li} \& \mathrm{Ma}$ [8].

\section{Application example : extrapolation of blazars from Fermi/LAT catalogues}

The Fermi/LAT collaboration recently released the 3FHL (The Third Catalog of Hard FermiLAT Sources) catalogue [9]. It contains 1558 sources detected above $10 \mathrm{GeV}$ in 84 months and analysed with the improved PASS8 analysis. The blazars dominate the sample of sources (78\%), followed by the galactic sources $(9 \%)$ and the unassociated ones $(13 \%)$. One of the main tasks of the extragalactic group of the CTA consortium, in the preparatory phase, is to estimate the number of detectable AGN by CTA, using as a starting point the 3FHL catalogue, and to measure its impact on population studies. A realistic analysis is available in [10].

To illustrate Gammapy capabilities, we studied the impact of adding an hypothetical exponential cut-off to the 3FHL Fermi/LAT spectra on the detection level. We selected a sample of 466 blazars, firmly identified or associated BL Lacs or flat spectrum radio quasars (FSRQs), having a redshift estimation in the catalogue and whose meridian transit occurs with zenith distance less than $30^{\circ}$ at least in one CTA site (north or south). We applied an exponential cut-off at energy $1 \mathrm{TeV} /(1+z)$ to the Fermi/LAT spectrum. To assess the detection level of a source, the following procedure was used :

- the spectrum is absorbed by the EBL model Dominguez et al. [5]

- the IRF labelled as " $5 \mathrm{~h}$ ", production $3 \mathrm{~b}$, are used according to the declination of the source ( $\delta \geq 0$ is north site)

- the simulation is done for $20 \mathrm{~h}$ of observation time

- the counts are integrated from $50 \mathrm{GeV}$ to $100 \mathrm{TeV}$

- the final significance is averaged on 20 simulations

The results of the simulations are shown on Figure 1. The fraction of sources detected above $5 \sigma$ is $\sim 53 \%$ (151/290) for BL Lacs and $\sim 10 \%$ (15/156) for FSRQs. Adding the cut-off decreases theses numbers to $46 \%$ and $8 \%$, showing the impact of the choice of the intrinsic spectral shape on detectability by CTA.

\footnotetext{
${ }^{2}$ This estimation has been realised with the Timit Python package and a $2.9 \mathrm{GHz}$ Intel Core i7 processor.
} 


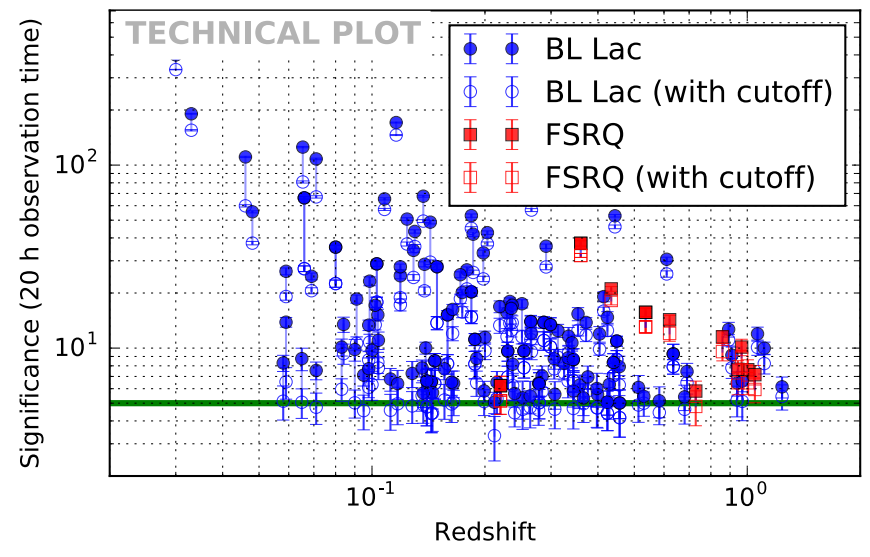

Figure 1: Average significance as a function of redshift for the simulated BL Lacs (blue circles) and FSRQs (red triangles). Full markers represent the 3FHL spectral model and empty markers represent the 3FHL spectral model multiplied by an exponential cut-off at $1 \mathrm{TeV} /(1+z)$.

\section{Application example: the EBL scale factor}

One of the main cosmological topics with the Cherenkov Telescope Array is to improve the constraints obtained with blazars on the diffuse extragalactic background light. A general and complete discussion can be found in [11]. In this section we will focus on the technical aspects to constrain an EBL scale factor for a given absorption model with Gammapy and the Python library Sherpa $^{3}$. Gammapy has been designed to provide flexibility in data-analysis to the end users. Simulations can be fed to widely-used tools that have been developed for X-ray astronomy, such as the Sherpa library, to produce higher data level. Sherpa gives access to the full-forward folding method to handle spectral reconstruction and provides a convenient way to do arithmetic with spectral models. Furthermore, each model can be adjusted on its own dataset and each parameter can be linked between different models, which makes the fit of the EBL scale factor straightforward. To obtain strong constraints on the EBL scale factor it is necessary to adjust multiple sources for different redshift ranges [11]. The choice of the modelling of the source will have an impact on our ability to reconstruct the EBL scale factor.

As an illustration, we studied the impact of an exponential cut-off, fixed at energy $1 \mathrm{TeV} /(1+$ $z$ ), on the determination of the EBL scale factor, called $\alpha$, for different redshifts. To proceed, we used the same sample of blazars described in [11] and adjusted for each source the intrinsic spectra $\phi_{\text {int }}$ and $\alpha$ resulting in an observed spectrum : $\phi(E)=\phi_{\text {int }}(E) \times e^{\left(-\alpha \tau_{m}(E, z)\right)}$ where $\tau_{m}$ is the $\gamma$-ray opacity of the intergalactic medium given by the model $m$. For each source, the following procedure was used to study the EBL scale reconstruction without (with) a cut-off :

- the intrinsic spectrum is given by the 3FHL catalogue, e.g. a power law or a log-parabola (and multiplied by a cut-off)

- the spectrum is absorbed by the EBL model [5]

\footnotetext{
${ }^{3}$ http://cxc.cfa.harvard.edu/contrib/sherpa/
} 


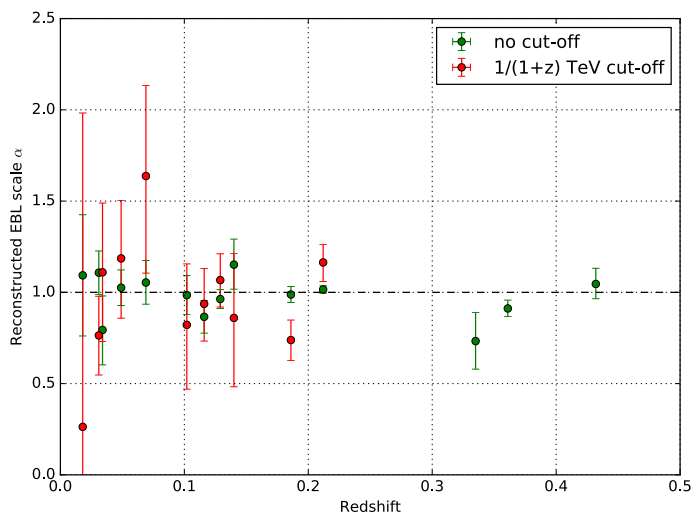

(a)

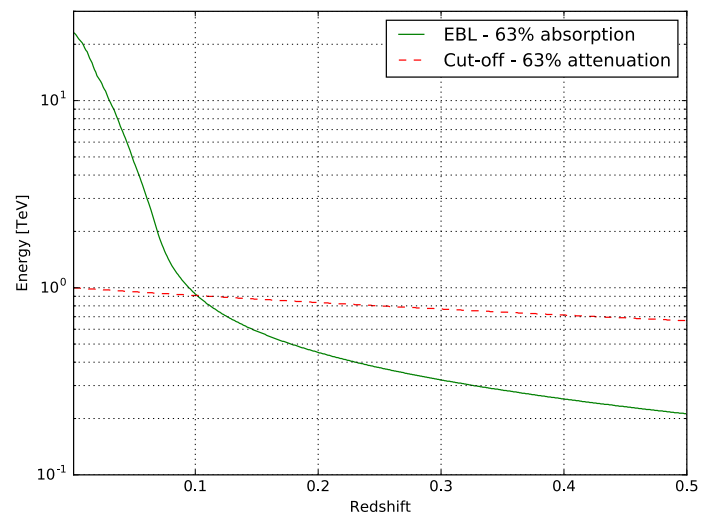

(b)

Figure 2: (a) Estimation of the reconstructed EBL scale $\alpha$ for individual sources used in [11] as a function of their redshift. The best estimation of $\alpha$ obtained without and with an additional cut-off on the intrinsic spectral model are respectively shown with green and red points. (b) The curves, displayed as a function of energy and redshift, corresponding to a loss of $63 \%$ of the photons are respectively shown in green and red for the EBL absorption and the exponential cut-off attenuation.

- the IRF labelled as " 5 h", production $3 b$, is chosen according to the declination of the source $(\delta \geq 0$ is north site)

- the simulation is done for $100 \mathrm{~h}$ of observation time

- the spectrum is fitted with the full-forward folding method in a fixed energy range from $50 \mathrm{GeV}$ to $5 \mathrm{TeV}$, with the intrinsic spectra model (and multiplied by a cut-off)

- the confidence interval bounds are computed for each parameter

We show on the technical Figure 2a, the reconstructed scale $\alpha$ as a function of the redshift of the sources for the two different spectral models. For low redshift, $z \leq 0.1$, the EBL absorption begins in the energy domain where the exponential cut-off is already present (see Figure $2 b$ ) so it makes it harder to constrain the EBL scale factor (the error bars increase). This could be in part compensated by a higher number of sources at low redshift. For the intermediate redshift range, typically from $z \sim 0.1$ to $z \sim 0.2$, the two effects are competitive and we are able to distinguish the two processes involved in the photon attenuation. At higher redshifts, the EBL extinction intervenes well before the exponential cut-off and the latter becomes less pertinent and thus under-constrained (for those cases, the maximisation likelihood procedure fails). That shows that Gammapy, in connection with the Sherpa library, is particularly well suited for these kinds of studies involving custom complex spectral models.

\section{Application example: observational window for $\gamma$-ray bursts}

$\gamma$-ray bursts are transient and explosive phenomena originating from cosmological distances. For the brief episode of emission their total energy output reaches $10^{51}-10^{53} \mathrm{ergs}$, placing them 
among the most powerful presently observed objects. A burst is characterised by a prompt phase dominated by X-ray and $\gamma$-ray photons (typically between $10 \mathrm{keV}$ and a few $\mathrm{MeV}$ ), lasting for a few milli-seconds to a few hundreds of seconds. It is followed by a second emission phase, called the afterglow, during which the electromagnetic emission is shifted to lower energies (Xrays, visible, radio) and rapidly decaying with time. The origin of the bursts is not fully understood. Nevertheless, the combination of the short variability timescale and the huge energy release suggest that GRBs are the results of cataclysmic events in the Universe, most likely associated with the births of stellar size black holes or rapidly spinning, highly magnetized, neutron stars. Up to now, the Fermi/LAT detected more than $100 \gamma$-ray bursts above $75 \mathrm{MeV}$, whereas IACTs never detected a significant $\gamma$-ray emission from a GRB. With its high effective area and its big field of view, CTA might detect a few of these events and help to better constrain their intrinsic properties. To get a more detailed picture about high energy and very high energy GRBs the reader may refer to [12] and [13], respectively.

Here, we used Gammapy to reproduce the result from [14] showing the detection significance of the bright $\gamma$-ray burst GRB $080916 \mathrm{C}$ as a function of the observational window. The spectral template is described as a power law of spectral index of 2 with an integrated flux in the $0.1 \mathrm{GeV}-$ $10 \mathrm{GeV}$ range at a time $t_{p}$ of $\Phi\left(t_{p}=6.5 \mathrm{~s}\right)=500 \times 10^{-5} \mathrm{~cm}^{-2} \mathrm{~s}^{-1}$. We further assume that the GRB is located at a distance ${ }^{4}$ corresponding to $z=3$. The flux decay over time is parameterized by a decay index $\delta=1.7$. In order to study the significance of the source as a function of the observational window, we defined 20 logarithmic time intervals representing the beginning and the end of the observations, from $20 \mathrm{~s}$ to 2 days. Starting from $t_{0}=20 \mathrm{~s}$ after the peak flux, the significance was computed for each physical logarithmic time interval in the following way :

- the average time interval $t_{\text {int }}$ is computed by taking into account the flux decay law

- the flux normalisation is computed for $t_{\text {int }}$

- the spectrum is absorbed with the EBL model from [5]

- the North IRF, labelled as " $0.5 \mathrm{~h}$ " from the production 2 are used

- the counts are integrated from $30 \mathrm{GeV}$ to $1 \mathrm{TeV}$

Results of the simulations are shown on Figure 3. The variation of the significance results from two effects, the flux decay of the source and a constant level of background as a function of time.

\section{Conclusion}

We have shown how Gammapy capabilities can be used to handle extragalactic science cases with the Cherenkov Telescopes Array. As mentioned in the introduction, we note that the Python scripts used to produce all results and figures presented in this contribution are available at https://github.com/gammapy/icrc2017-gammapy-cta-egal. This means anyone can reproduce and check the results, and use the scripts as examples to start their own studies.

\footnotetext{
${ }^{4}$ The measured redshift of GRB $080916 \mathrm{C}$ is $z=4.35 \pm 0.15$ [15].
} 


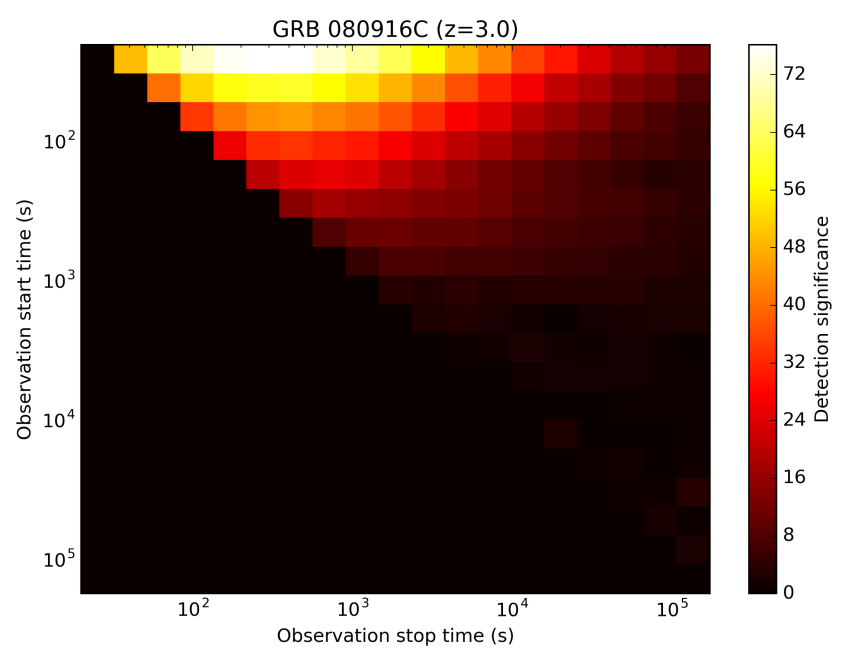

Figure 3: Significance of detection as a function of time windows (t-start, t-stop) for the template of GRB as described in the text.

\section{Acknowledgments}

This work was conducted in the context of the CTA Consortium. Julien Lefaucheur would like to thank Régis Terrier (APC/CNRS) for useful discussions about data analysis with the Python library Sherpa.

\section{References}

[1] A. Zech, M. Cerruti and D. Mazin, Expected signatures from hadronic emission processes in the TeV spectra of BL Lacertae objects, A\&A 602 (Feb., 2017) A25, [1703.05937].

[2] C. Deil et al., Gammapy - A prototype for the CTA science tools, in The 35th International Cosmic Ray Conference, Proceedings of science, 2017.

[3] L. Jouvin et al., Toward a 3D analysis in Cherenkov $\gamma$-ray astronomy, in The 35th International Cosmic Ray Conference, Proceedings of science, 2017.

[4] M. Gernot et al., Performance of the Cherenkov Telescope Array, in The 35th International Cosmic Ray Conference, Proceedings of science, 2017.

[5] A. Domínguez et al., Extragalactic background light inferred from AEGIS galaxy-SED-type fractions, MNRAS 410 (Feb., 2011) 2556-2578, [1007 . 1459].

[6] A. Franceschini et al., Extragalactic optical-infrared background radiation, its time evolution and the cosmic photon-photon opacity, A\&A 487 (Sept., 2008) 837-852, [0 805.1841 ].

[7] J. D. Finke et al., Modeling the Extragalactic Background Light from Stars and Dust, ApJ 712 (Mar., 2010) 238-249, [0 905 .1115]. 
[8] T.-P. Li and Y.-Q. Ma, Analysis methods for results in gamma-ray astronomy, ApJ 272 (Sept., 1983) 317-324.

[9] The Fermi-LAT Collaboration, 3FHL: The Third Catalog of Hard Fermi-LAT Sources, ArXiv e-prints (Feb., 2017), [1702.00664].

[10] T. Hassan et al., Extragalactic source population studies at very high energies in the Cherenkov Telescope Array era, in The 35th International Cosmic Ray Conference, Proceedings of science, 2017.

[11] F. Gaté et al., Studying cosmological gamma-ray propagation with the Cherenkov Telescope Array, in The 35th International Cosmic Ray Conference, Proceedings of science, 2017.

[12] F. Piron, Gamma-ray bursts at high and very high energies, Comptes Rendus Physique 17 (June, 2016) 617-631, [1512.04241].

[13] S. Inoue and other, Gamma-ray burst science in the era of the Cherenkov Telescope Array, Astroparticle Physics 43 (Mar., 2013) 252-275, [1301.3014].

[14] E. Bissaldi et al., Prospects for Gamma-Ray Burst detection by the Cherenkov Telescope Array, in 11th Workshop on Science with the New generation of High Energy Gamma-ray Experiments, Il Nuovo Cimento C, 2017.

[15] A. A. Abdo et al., Fermi Observations of High-Energy Gamma-Ray Emission from GRB 080916C, Science 323 (Mar., 2009) 1688. 